\title{
Para una nueva edición de las Poesías de Miguel de Unamuno: La torre de Monterrey a la luz de la luna
}

\author{
Assunta Claudia Scotto di Carlo \\ Universidad Telemática eCampus
}

Título: Para una nueva edición de las Poesías de Miguel de Unamuno: La torre de Monterrey a la luz de la luna.

Resumen: Este artículo se propone presentar la edición filológica de una de las composiciones de Poesías - la primera colección de poemas publicada por Miguel de Unamuno en 1907-; en concreto la que lleva por título La torre de Monterrey a la luz de la luna. La elección de esta obra fue dictada por varios factores, el principal la variedad de materiales disponibles, hecho que la dota de interés para la construcción del aparato. En la última parte del artículo se ofrece un breve comentario al texto y algunas consideraciones sobre el uso del aparato.

Palabras clave: Unamuno, Poesias, Edición filológica, Monterrey, Aparato de variantes

Fecha de recepción: 12/3/2014.

Fecha de aceptación: 1/6/2014.
Title: For a New Edition of Miguel de Unamuno's Poesías: La torre de Monterrey a la luz de la luna.

Abstract:This article aims to present the philological edition of one of the poems of Poesias, the first collection of poems published in 1907 by Miguel de Unamuno, named La torre de Monterrey a la luz de la luna. Choosing this poem is due to several reasons, above all the variety of available materials which makes it interesting for the construction of the apparatus. The last part of this article provides a brief commentary to the text and some consideration about the apparatus.

Key words: Unamuno, Poesías, Philological edition, Monterrey, Apparatus of variants.

Date of Receipt: 12/3/2014.

Date of Approval: 1/6/2014. 


\section{i. Las Poesías de Unamuno}

En 1907 se publica la primera colección de poemas de Miguel de Unamuno, titulada Poesías. El trabajo se divide en 16 secciones de diferente extensión para un total de 102 composiciones ${ }^{1}$. Los textos, que el propio Unamuno en el primer trabajo define como "de otoño" para aludir a la edad tardía en que los publica, fueron compuestos en realidad durante un periodo de tiempo muy extenso: si bien en los primeros ańos del siglo XX observamos un aumento significativo de su producción poética, hay, en cambio, textos que fueron escritos mucho antes, como El Árbol solitario que, según apuntó el propio escritor en $M i$ primer artículo ${ }^{2}$, fue publicado en 1880 en El Noticiero Bilbaino.

La continua labor de escritura poética se entrelaza, por tanto, en la actividad de Unamuno con la composición de textos como Amor y Pedagogía, la Vida de Don Quijote y Sancho y Recuerdos de niñez y de mocedad, y esta duplicidad afecta en muchos casos al estilo de la prosa, que tiende a convertirse en "poética", al absorber las estructuras formales propias de la lírica, como son las cláusulas rítmicas o el recurso a principios retóricos propios de la poesía. Desde este punto de vista, un estudio de los aspectos estilístico-formales de este texto puede proporcionar resultados útiles que arrojen luz sobre el horizonte más amplio de la producción de Unamuno, quien, de alguna manera, enfoca su pensamiento ora hacia la poesía ora hacia la prosa, oscilando del ensayo hacia un plano más creativo, para plasmar de este modo sus reflexiones en diferentes géneros literarios.

Con esta primera colección, además, Unamuno rompe abiertamente con la tradición contemporánea española que, a sus ojos, tenía la limitación de ser poco variada en sus formas y, sobre todo, de estar demasiado atada a algunas cadencias rítmicas consideradas vacías y banales. En contraste con las modas poéticas de la época y sobre todo con el Modernis-

1 Analizando el índice de la primera edición del volumen se pueden contar, sin embargo, solamente 100 poemas. No se trata de añadidos sucesivos: los dos poemas que faltan, Al sueño y La sacerdotisa, forman ya parte de la colección, pero no están incluidos en el índice.

2 Miguel de Unamuno, "Mi primer artículo", en Obras Completas, ed. Manuel García Blanco, Madrid, Escelicer, 1966, VIII, pp. 520-522. 
mo, Unamuno trata de crear una nueva forma de escritura y, para ello, dirige su atención a los metros italianos (especialmente al verso libre) y a los “musings" ingleses: Leopardi, Carducci, Wordsworth, Coleridge y Tennyson, junto con Maragall, se convierten en las principales referencias para su escritura poética:

Tengo la pretensión de que mi poesía aporta algo a las letras espańolas de hoy. En su forma es casi toda, no toda, al modo del verso libre italiano, y el resto en romance endecasílabo. En cuanto al fondo se parece a los musings ingleses, a la poesía meditativa inglesa, la de Wordsworth, Coleridge, Browning, etc. ${ }^{3}$

A pesar de su importancia, tanto dentro del panorama literario español, como en la producción literaria del autor, se echa en falta todavía una edición crítico-filológica de esta colección que tenga en cuenta los diferentes materiales, tanto manuscritos como impresos, y arroje luz sobre el decurso gradual y la transformación de las estructuras poéticas.

El libro de Manuel García Blanco Don Miguel de Unamuno y sus poesías, publicado en 1956, proporciona información importante acerca de los materiales de archivo conservados en la Casa-Museo de Unamuno de Salamanca, vinculables con la colección de poemas de 1907. Sin embargo, este estudio, que asume principalmente la forma de un catálogo anotado, es parcial, ya que no considera todo el material preparatorio y se basa principalmente en el manuscrito autógrafo 62/2 (en el aparato crítico y en las páginas siguientes indicado como $m s^{a}$ ); el cual, a pesar de ser incompleto y de estar lleno de lagunas, ocupa, como se verá, una posición central en el sistema de los documentos que dan testimonio de una de las últimas y decisivas etapas de desarrollo de la colección. Este documento de gran valor, con sus 69 poemas, nunca ha sido estudiado ampliamente en su intrínseco valor filológico-textual, sino que se utiliza casi exclusivamente como una fuente para la datación de los poemas individuales.

Durante la investigación que llevé a cabo en los archivos, he podido verificar la presencia, junto al manuscrito $m s^{a}$, de un número elevado

3 Carta a P. Jiménez Ilundain, en Miguel de Unamuno, Epistolario Americano, ed. Laureano Robles, Salamanca, Universidad de Salamanca, 1996, pp. 63-64. 
de redacciones y versiones manuscritas de cada uno de los poemas. Este material se enriquece con la correspondencia de Unamuno: se sabe efectivamente que solía enviar sus obras a algunos amigos y conocidos para que las leyeran (entre los nombres destacan Azorín y Maragall), y que de ellos recibía consejos, opiniones y reflexiones que a menudo influían en la elaboración final. Otra fuente la constituyen las revistas en las que Unamuno publicó algunos de los versos que luego incluyó en Poesías.

En este breve artículo me gustaría presentar la edición filológica de una de las composiciones de Poesias, incluida en la sección Castilla y titulada La torre de Monterrey a la luz de la Luna. La elección de este poema fue dictada por varios factores, el primero de todos, como se verá inmediatamente, por la variedad de materiales disponibles, hecho que lo hace interesante para la construcción del aparato crítico. En segundo lugar, me parece que el uso de la estructura métrica de la estrofa sáfica lo convierte en un ejemplo de la experimentación métrica de Unamuno y de la influencia que ejerció sobre él la poesía italiana de la época, especialmente la de Carducci. La crítica está bastante dividida sobre la huella real de Carducci en la elección de este molde estrófico que, gracias a los modelos italianos, se había difundido en España ya desde la primera mitad del siglo XVI y, por tanto, dicha elección podría valorarse como el resultado de una "marcada intención clasicista" ${ }^{4}$. No obstante, debe recordarse que, a partir de 1904, cuando se dedicó a la traducción de Miramare, Unamuno comenzó a experimentar con la estrofa sáfica y que en numerosas ocasiones declaró que seguía el patrón de Carducci: "En mis Poesías verá muchos sáficos al modo carducciano"s.

La torre de Monterrey a la luz de la Luna se encuentra dentro del $m s^{a}$ y se conserva también en una versión manuscrita en la caja 76/115 (indicada en el aparato crítico como $\left.m s^{l}\right)$. Este documento, $m s^{l}$, que probablemente representa una de las primeras fases de elaboración, se compone de un sobre y de un bifolio: el primero, cuya escritura parece coincidir con la de García Blanco, contiene el título del poema, el nombre de la colección donde está

4 Véase Vicente González Martín, La cultura italiana en Miguel de Unamuno, Salamanca, Universidad de Salamanca, 1978, pp. 182-184, en la que el estudioso reconstruye el debate crítico sobre el tema.

5 Miguel de Unamuno, Epistolario Americano, pp. 277-278. 
incluido, el año, el número (10) que ocupa en Poesías escrito a lápiz rojo y, en la parte inferior, la antigua catalogación de archivo (1.2/359). El bifolio, autógrafo de Unamuno, contiene el texto en los fols. $1 \mathrm{r}$ y $1 \mathrm{v}$.

Los manuscritos no presentan ninguna datación, aunque gracias a la correspondencia sabemos que la composición fue escrita en 1906: en una carta de 13 de julio de ese año, efectivamente, Everett Olmsted, el mismo a quien Unamuno dedica la sección de los Salmos en Poesías, hace referencia a una lectura suya de este poema. Sin embargo, se puede retrotraer la composición a unos meses antes gracias a una carta, señalada por primera vez en el estudio de García Blanco, que Unamuno envió en mayo de 1906 a Federico de Onís:

He avanzado bastante en mi Tratado del Amor de Dios; pero sobre todo hago versos. En lo que va de año llevo hechos tantos casi como en el resto de mi vida. Y, para acabar, aquí van los últimos. "La torre de Monterrey a la luz de la luna" ${ }^{6}$.

Las cartas nos permiten no solo obtener información útil para fechar esta composición, sino también para deducir otros datos sobre el modo de redacción característico de Unamuno, que consiste, como ya hemos dicho, en enviar sus textos a algunos amigos para que los lean. Esa misma carta a Federico de Onís, en 1988, ha sido publicada por Carlos William de Onís en Miguel de Unamuno en su Salamanca ${ }^{7}$. Puesto que no me ha sido posible analizar el texto autógrafo enviado por Unamuno a Federico de Onís, en el aparato he añadido a las variantes recogidas por García Blanco en su estudio, identificadas con la sigla $G B$, las que se documentan en la obra de C. W. de Onís, identificadas con la sigla FO. Esta operación plantea, claro está, algunos problemas metodológicos debidos al uso de materiales «de segunda mano» en lugar de las cartas autógrafas: en este caso se trata de utilizar dos transcripciones distintas de un mismo documento. Sin embargo, sería quizás mucho más grave renunciar a valorar, siempre

6 Manuel García Blanco, Don Miguel de Unamuno y sus poesias, Salamanca, Universidad de Salamanca, 1954, p. 78.

7 Federico de Onís, Unamuno en su Salamanca, ed. Carlos William de Onís, Salamanca, Universidad de Salamanca, 1988. pp. 46-47. 
con la debida prudencia, unos testimonios tan interesantes, excluyendo de este modo las variantes señaladas en el único estudio que hasta el momento se ha interrogado sobre las diversas redacciones de las composiciones de Unamuno o sobre las variantes que se localizan en la única obra que recopila todo el epistolario entre el rector de Salamanca y Onís.

El texto fue publicado el 19 de agosto de 1906 en La Publicidad de Barcelona (indicado en el aparato como Pub), junto con Elegía en la muerte de un perro, Duerme alma mía, En la muerte de un hijo y En la catedral vieja de Salamanca; este artículo es muy interesante, porque representa una prueba más de la fina trama que une la labor de escritura simultánea de varios textos, a pesar de ser tan diferentes los unos de los otros. Los cinco poemas, recogidos bajo el título Poesías inéditas de Unamuno, se incluyen en un extenso artículo (Nuevos Libros de Unamuno), firmado por Luis de Zulueta, que se centra en la última producción poética y literaria de Unamuno. Además de la poesía, en este artículo de corte documental, Zulueta también presenta fragmentos de los Recuerdos de niñez y de mocedad y del Tratado de Amor de Dios ${ }^{8}$.

El estudio de las variantes nos permite identificar el $m s^{l}$ como el texto más antiguo; a continuación, vienen GB (mayo de 1906), Pub (agosto de 1906) y, finalmente, $m s^{a}$. Particularmente significativa es la variante del v. 1 «robusta»

8 Este artículo, por lo tanto, es interesante por dos motivos: por un lado, permite identificar algunas variantes en las poesías; y, en segundo lugar, nos brinda la posibilidad de aclarar algunos detalles relevantes con respecto a la elaboración de Recuerdos, que ya comenté en mi estudio sobre la obra: véase Assunta Claudia Scotto di Carlo, "Il vissuto e il narrato". Los "Recuerdos de niñez y de mocedad" de Miguel de Unamuno, Pisa, Ets, 2012. En el artículo de Zulueta, donde se publica el capítulo quince de la primera parte de Recuerdos, el título de la obra es Memorias de niñez y de mocedad, confirmando así la indecisión entre el término Recuerdos y el término Memorias que ya había surgido en las cartas. Gracias a la breve introducción de Zulueta, también es posible afirmar que en agosto de 1906 el texto estaba listo, por lo que el autor puede escribir: "Las «Memorias de niñez y de mocedad", ya terminadas, forman un volumen corto, interesante y divertido". El texto publicado en 1906 presenta algunas variantes en comparación con el publicado en 1908: [186] al Instituto] en el Instituto; [189] en Archanda] en la cordillera de Archanda; [191] de modo] de noche; [194] como diré] como diré adelante; formándose famosas] formándose entonces famosas; [197] lágrimas y vítores] vítores y lágrimas. Estas variantes pueden ser integradas en la edición sinóptica que ofrezco en el libro que acabo de mencionar. 
utilizada en $m s^{I}$, en GB y en $P u b$, pero que se corrige por "cuadrada» (que es la lección publicada en Poesías en 1907) solo en $m s^{a}$. La fecha de publicación de la composición en la revista Publicidad (el 19 de agosto de 1906) establece el término post quem para la finalización del manuscrito $m s^{a}$ : esto confirma la importancia de este documento, que hay que atender no solo como testimonio del último estadio evolutivo del texto de La torre de Monterrey a la luz de la luna, sino también de una de las últimas etapas de la organización de la colección que, de hecho, se publicó unos pocos meses más tarde.

\section{Aparato}

La edición crítica de un texto considerado en su lenta constitución a lo largo de un eje cronológico que se percibe como coherente y dinámico es un acto interpretativo distinto cada vez. Una de las principales razones es la diferencia de los materiales disponibles a los que se ańade la falta de un sistema de criterios y signos compartidos?.

Esta diversidad implica inevitablemente algunas repercusiones en la elaboración del aparato crítico de las variantes, que deben representar de manera clara y concisa todo el proceso de corrección.

La falta de un sistema compartido contribuye a hacer esta operación todavía más complicada. En el caso de la poesía de Unamuno el tipo de material disponible, compuesto como se ha visto por manuscritos, cartas, publicaciones en revistas y, en algunos casos, por transcripciones hechas por diversas manos, me ha llevado a elegir entre los diferentes modelos posibles de referencia en relación con la poesía del siglo XX, el propuesto por Dante Isella en la edición de las Poesías de Vittorio Sereni, en el que el estudioso ha examinado, con vistas al trabajo ecdótico y filológico-hermenéutico, un conjunto de materiales muy similares. Sin embargo, la especificidad de la

9 Dante Isella, "Ancora della filología d'autore", en Le carte mescolate vecchie e nuove, Torino, Einaudi 2009, p. 239. Véase además AA. VV., Crítica genética y edición de manuscritos hispánicos contemporáneos. Aportaciones a una "poética de transición entre estados", eds. Bénédicte Vauthier y Jimena Gamba Corradine, Salamanca, Universidad de Salamanca, 2012; y Paola Italia, Editing Novecento, Roma, Salerno Editrice, 2013. 
escritura de Unamuno y la voluntad de facilitar la consulta del aparato me han llevado a introducir criterios diferentes, más centrados en la reconstrucción y la representación del proceso de corrección del texto ${ }^{10}$.

El aparato consta de 4 secciones. En la primera se encuentran los datos que tienen relación con la colocación del poema dentro de la primera edición de la colección:

POE 35-37 (II, 4) significa que la composición se encuentra en Poesías en las pp. 35-37 y que es la cuarta composición de la segunda sección de la obra.

En la segunda franja se enumeran y se comentan brevemente los testimonios manuscritos del texto, mientras que en la tercera se encuentran las referencias a las publicaciones en los periódicos.

El cuarto nivel contiene el aparato de las variantes y se organiza de acuerdo con los siguientes criterios:

- Dada la consistencia de la tradición, contenida pero suficientemente representativa de las tipologías de los materiales y de las modalidades de elaboración textual desarrolladas por Unamuno, se ofrece un aparato positivo, enriquecido por algunas indicaciones útiles a la hora de reconstruir las fases genético-evolutivas del texto. Para cada variante se pone en la base, precedida por el número de la estrofa y del verso y seguida por un corchete de cierre, la lección definitiva recogida en Poesías de 1907. De esta lección se puede seguir la dinámica en dirección evolutiva, puesto que las indicaciones de los testimonios de referencia, identificados por una sigla en cursiva, se ofrecen en orden cronológico hasta la última, que está representada casi siempre por $m s^{a}$. Cada una de las correciones se representa de manera unitaria, describiendo entre paréntesis de manera sintética, pero clara, en su fenomenología.

- Las variantes se acompañan de algunas indicaciones en cursiva: interl. sup. (interlínea superior); interl. inf. (interlínea inferior); marg. inf. (escrito en el margen inferior del folio).

10 Paola Italia, Giulia Raboni, Che cosè la filologia d'autore, Roma, Carocci, 2010, p. 9. 
- Los signos diacríticos var alt. $<$ > encierran las variantes alternativas; dichas variantes van separadas por el signo vertical $\mid$.

- Los paréntesis angulares > < encierran las tachaduras inmediatas (es decir, las que se realizan dentro de una misma fase redaccional).

- En las sustituciones presumiblemente tardías, el subrayado evidencia las palabras que se tachan luego en la fase sucesiva.

- En el caso en que en un manuscrito sea posible seguir su dinámica evolutiva concreta los exponentes alfabéticos (en cursiva) que preceden a cada uno de los niveles de corrección indican su sucesión.

- Las variantes (o secuencias de corrección) de diversos testimonios aparecen separados por un punto y coma.

- Cuando los testimonios coinciden con el texto base se utiliza la sigla T.

\section{La torRe De Monterrey a La LUZ DEL LUNA: TeXTO ${ }^{\text {II }}$ y Aparato}

\section{LA TORRE DE MONTERREY}

A LA LUZ DE LA LUNA

Torre de Monterrey, cuadrada torre, que miras desfilar hombres y días, tú me hablas del pasado y del futuro Renacimiento.

De día el sol te dora y a sus rayos se aduermen tus recuerdos vagarosos, te enjabelga ${ }^{12}$ la Luna por las noches,

11 Reproduzco aquí el texto de la edición de 1907, aunque he preferido aplicar las reglas de acentuación actuales.

12 La edición del 1907, al igual que todos los testimonios manuscritos e impresos unamunianos (véase el aparato crítico), utilizan la forma enjabelga, para la actual enjalbega. El término no está atestiguado en el Nuevo tesoro lexicográfico de la lengua española (NTLLE) ni tampoco en el CORDE, pero en el Diccionario crítico etimológico castellano e hispánico por J. Corominas y J. A. Pascual viene señalado como caso de metátesis salmantina. Los editores de Poesías a partir de García Blanco eligen enjalbega, mientras que, en mi caso, prefiero la lectura enjabelga, por ser peculiar del léxico de Unamuno; por la misma razón conservo la forma inconciencia en el v. 12. 
y se despiertan.

Velas tú por el día, enajenada, confundida en la luz que en sí te sume $y$ en las oscuras noches te sumerges en la inconciencia.

Mas la Luna en unción dulce al tocarte despiertas de la muerte y de la vida, y en lo eterno te sueñas y revives en tu hermosura.

¡Cuántas noches, mi torre, no te he visto a la unción de la Luna melancólica despertar en mi pecho los recuerdos de tras la vida!

De la Luna la unción por arte mágica derrite la materia de las cosas y su alma queda así flotante y libre, libre en el sueño.

Renacer me he sentido a tu presencia, torre de Monterrey, cuando la Luna de tus piedras los sueńos libertaba y ellas cedían.

Y un mundo inmaterial, todo de sueño, de libertad, de amor, sin ley de piedra, mundo de luz de luna confidente soñar me hiciste.

Torre de Monterrey, dime, mi torre, tras de la muerte el Sol brutal se oculta o es la Luna, la Luna compasiva, del sueño madre?

¿Es ley de piedra o libertad de ensueño lo que al volver las almas a encontrarse 
las unirá para formar la eterna

torre de gloria?

Torre de Monterrey, soñada torre,

que mis ensueños madurar has visto, tú me hablas del pasado y del futuro

Renacimiento.

POE 35-37 (II, 4)

Se conserva una versión manuscrita en un bifolio ( $m s^{l}$ fols. 1r y $1 \mathrm{v}$ ) cuyas estrofas están numeradas a lápiz por Unamuno. El f. $2 \mathrm{r}$ está en blanco. En el f. $2 \mathrm{v}$ se encuentra, también a lápiz, la indicación de un nombre acompañado por la indicación del pueblo (se puede suponer que se trata de un conocido, o incluso del destinatario del texto) "Jesús García Sanchez (Cabezuela)», y la vieja catalogación del archivo “1.2/359”. En el f. 1r, además, hay algunas líneas trazadas a lápiz rojo y que forman un ángulo recto. Fue enviada en una carta, por ahora ilocalizable, a Francisco de Onís (las variantes señaladas por García Blanco, que declara haber visto el documento, se indican con la sigla $G B$, las variantes que se encuentran en el epistolario publicado por William Carlos de Onís se indican con la sigla $F O$ ). Una segunda versión se encuentra en $m s^{a}$ (ff. 54-57; ff. 54-56bis, según la numeración de Unamuno situada en la esquina superior derecha, también a lápiz, excepto la hoja 56bis que está escrita a pluma). En f. 54, junto al título, aparece una numeración distinta a lápiz (39) del propio Unamuno, que quizás indique una ordenación distinta de las poesías. Las estrofas están separadas por un trazo horizontal a lápiz.

Se publica en La Publicidad, Barcelona, 19 de agosto de 1906 (Pub).

I

v. 1 cuadrada] robusta $m s^{l} G B F O$ Pub; ${ }^{a}$ robusta ${ }^{b}$ cuadrada $m s^{a}$

v. 4 Renacimiento.] Renacimiento! $m s^{1}$; T FO Pub $m s^{a}$

II

v. 5 sol] Sol FO Pub; T $m s^{1} m s^{a}$

v. 7 enjabelga] enjalbega $G B$; T $m s^{l} F O$ Pub $m s^{a}$ 
por las noches,] por las noches FO; T $m s^{1} F O P u b m s^{a}$

v. 8 se despiertan] se despierta $G B$; T $m s^{l} F O P u b m s^{a}$

\section{III}

v. 10 que en sí te sume] var alt qque así ${ }^{a}$ se absorve ${ }^{b}$ te absorve | que en sí te sume (interl. sup.; sume también con lápiz interl. inf.), $m s^{1}$ (las vocales o $y$ e de absorve subrayadas y conectadas con una linea a las vocales correspondientes de noches al v. 7); T Pub ms (sume, FO)

\section{IV}

v. 13 Más la Luna en unción dulce al tocarte] ${ }^{a}$ Mas al tocarte con su unción la luna ${ }^{b}$ Mas al tocarte en dulce unción la luna ${ }^{c}$ Mas la luna en unción dulce al tocarte (Unamuno olvida tachar en dulce de la fase anterior) $m s^{l}$; unión $F O$; $\mathbf{T}$ Pub $m s^{a}$

v. 16 hermosura] ${ }^{a}$ belleza ${ }^{b}$ hermosura $m s^{l}$; T FO Pub $m s^{a}$

$\mathrm{V}$

v. 17 noches] veces $G B$; T $m s^{1} F O$ Pub $m s^{a}$

v. 18 a la unción de la] >á luz de la $</$ a la unción de la (variante inmediata escrita en la linea siguiente) $m s^{1}$; T FO Pub ms

v. 19 recuerdos] ${ }^{a}$ fantasmas ${ }^{b}$ recuerdos $m s^{l}$; T FO Pub $m s^{a}$

VI

v. 21 De la luna] ${ }^{a}$ De $m i$ luna ${ }^{b}$ De la luna (la con lápiz sobre mi; La luna escrito también en la interl. sup.) $m s^{1}$; T FO Pub ms ${ }^{a}$

por arte mágica] var alt. por arte mágica | á nuestros ojos` $m s^{l}$; T FO Pub $m s^{a}$

v. 22 cosas] cosas, FO; $\mathbf{T} m s^{1}$ Pub $m s^{a}$

v. $23 \mathrm{su}{ }^{a} \underline{\mathrm{el}}^{b}$ su $m s^{1}$; T Pub $m s^{a}$

así flotante] $\mathbf{T} m s^{1} F O$; así, flotante Pub $m s^{a}$

VII

v. 26 Luna] luna $m s^{l}$; ${ }^{a}$ luna ${ }^{b}$ Luna $m s^{a}$; T FO Pub

v. 28 ellas] ellos $G B$; $\mathbf{T} m s^{1} F O$ Pub $m s^{a}$

cedían] ${ }^{a}$ morían ${ }^{b}$ cedían $m s^{l}$; T FO Pub $m s^{a}$

VIII

v. 29 un mundo] el mundo $G B$; mundo FO; $\mathbf{T} m s^{1}$ Pub $m s^{a}$ sueño,] sueño $F O$; $\mathbf{T} m s^{l}$ Pub $m s^{a}$

v. 31 confidente] compasiva GB FO; $\mathbf{T} m s^{1} \mathrm{Pub} m s^{a}$ 
v. 32 soñar me hiciste] soñarme hiciste $G B$; $\mathbf{T} m s^{l} F O P u b m s^{a}$

IX

v. 34 tras] ¿tras FO; $\mathbf{T} m s^{1}$ Pub $m s^{a}$

v. 35 Luna, la Luna] a luna, la luna ${ }^{b}$ Luna, la Luna $m s^{1}$; T FO Pub $m s^{a}$

v. 36 del sueño madre] ${ }^{a}$ madre del sueño ${ }^{b}$ del sueño madre $m s^{l}$; T $F O$ Pub $m s^{a}$

$\mathrm{X}$

vv. 39-40 la eterna / torre de gloria] " la torre / torre de gloria ${ }^{b}$ la torre / de eterna gloria cla torre / var alt de fe y gloria | de paz y gloria) (de fe y con lápiz; paz y con lápiz en el marg. inf.) deterna / torre de gloria (Unamuno olvida tachar paz y de la fase anterior) $\mathrm{ms}^{1}$; T Pub $m s^{\mathrm{a}}$ (eterna $G B F O$ )

XI

v. 41 soñada torre] torre soñada (la $\mathrm{t}$ de torre sobre una s; posiblemente Unamuno iba a escribir soñada torre) $m s^{1}$; T FO Pub $m s^{a}$

v. 42 madurar] ${ }^{a}$ desfilar ${ }^{b}$ renacer (la variante se tuvo que escribir antes con lápiz en el marg. derecho y luego con tinta en la interl. sup.) ms ${ }^{1}$; T FO Pub ms ${ }^{a}$

\section{Algunas consideraciones sobre el uso del aparato}

El estudio del aparato ofrece algunos puntos interesantes de reflexión a diferentes niveles ${ }^{13}$. En primer lugar, el continuado trabajo de lima sobre el texto, al cual Unamuno regresa una y otra vez incluso para intervenciones minúsculas, y que atestiguan una atención profunda a los aspectos formales del texto durante todas sus fases de construcción y revisión. Ofrezco solo un ejemplo de esta atención -que vale la pena señalar por su peculiar y minuciosa actividad de elaboración- en las líneas de guiones que unen los versos 7 y 10 (véase el aparato del v. 10). Me parece que estos ligeros trazos gráficos permiten constatar y sacar a la luz, a través de un

13 Muchas de las variantes de $G B$ parecen lecturas erróneas del copista. Las pocas lecciones singulares de Pub se deben seguramente al tipógrafo. En rigor, en ninguno de los dos casos se trata de variantes de autor. 
detalle pequeño pero evidente, una de las cuestiones que más preocupan a principios de siglo a Unamuno, poeta de oído refinado gracias a su largo estudio de la literatura griega: la cuestión de la musicalidad, o más bien de la excesiva atención al ritmo, entendido como cadencia, como flujo articulado y armónico de la poesía.

Probablemente, al volver a leer el texto después de una primera redacción, Unamuno no estuvo muy convencido del verbo "absorve" (pero sobre esto volveremos a continuación), que además establece una relación de asonancia con la palabra "noches": con líneas de guioncitos conecta las vocales de los dos términos para poner de relieve este fenómeno. Ese vínculo sonoro entre dos versos, amplificado por la repetición de la palabras "noches" también en el v.11, no convencía del todo al escritor que, de hecho, en $m s^{l}$ anota inmediatamente a lápiz, "sume". Posteriormente reflexiona sobre una forma alternativa del verso basada en el nuevo verbo, sin elegir todavía entre las dos posibilidades. La elección, sin embargo, tendrá lugar en redacciones posteriores, donde todas concuerdan ya en proponer la lectura «sume», con la cual se elimina la asonancia, que hubiera sido la única entre dos versos tan cercanos. Pero no se pueden pasar por alto las consecuencias de esta elección que, para eliminar una asonancia, crea una repetición mucho más fuerte (no sólo de sonido, sino también de sentido) entre dos versos sucesivos que se cierran, respectivamente, con "sume" y con "sumerges". Para comprender plenamente la operación de Unamuno, es necesario un doble ejercicio: antes que nada, hay que entender la posición del escritor sobre la relación entre poesía y música durante aquellos años $\mathrm{y}$, a continuación, hay que interpretar su elección teniendo en cuenta el significado de todo el poema.

Las pequeñas líneas verticales que el autor traza en su manuscrito son el indicio concreto de la plasmación en la práctica de escritura de la larga meditación teórica de Unamuno sobre los temas del ritmo y la musicalidad.

La decisión de reducir las conexiones sonoras entre los versos y las palabras, además, es plenamente coherente con las numerosas declaraciones hechas por Unamuno a lo largo de aquellos mismos años en contra de la utilización de todos los recursos formales y retóricos que se utilizan para dar al texto un ritmo y una cadencia superficial: el más 'bárbaro' de los 
artificios es, sin duda, la rima ${ }^{14}$. Su negativa a seguir las principales tendencias de principios de siglo impulsó varias veces a Unamuno a expresar opiniones amargas hacia una poesía reducida a un ejercicio puramente formal ${ }^{15}$, muy poco atenta a los valores semánticos y ético-ideológicos, vacía, inconsistente en cuanto al contenido. Aun en Poesías, en los versos del célebre Credo Poético, verdadero manifiesto de su poética ${ }^{16}$, argumenta sin ambages contra esta tendencia, que define como explícitamente 'musical':

Peso necesitan, en las alas peso,

la columna de humo se disipa entera,

algo que no es música es la poesía,

la pesada solo queda ${ }^{17}$. (vv. 5-8)

El rechazo de la musicalidad no debe interpretarse, por supuesto, como una renuncia a la búsqueda de la armonía del texto; lo que Unamuno critica es la reducción trivial de la poesía a la musicalidad. Incluso en el caso de los versos del Credo Poético sería necesario recurrir al estudio de los manuscritos: al analizar el manuscrito conservado en la caja 76/113, que contiene uno de los primeros borradores del texto, se comprueba que, al llegar al v. 7, Unamuno escribe "algo más"; a continuación elimina el "más" y escribe el resto del verso tal como lo conocemos hoy los lectores.

14 El término "bárbaro artificio" en referencia a la rima es utilizado por Unamuno en una carta dirigida a Francisco Antón y publicada en José Ignacio Tellechea Idígoras, "Unamuno y F. Antón Casaseca. Epistolario", Cuadernos de la Cátedra Miguel de Unamuno, 36 (2001), págs. 145-276; González Martín reconstruye brevemente el cambio gradual de actitud hacia la rima que llevará a Unamuno, alrededor de 1909, a aceptar el concepto carducciano de rima generadora. Véase Vicente González Martín, op. cit., pp. 177-198.

15 Los ataques se dirigen sobre todo a los poetas modernistas; véase, entre otros, Francisco Javier Díez de Revenga, "Unamuno ante la poesía y los poetas modernistas: reacciones y controversias", en El joven Unamuno en su época: Actas del Coloquio Internacional Würzburg 1995, eds. Theodor Berchem, Hugo Laitenberger, Salamanca, Junta de Castilla y León, 1997, pp. 307-318.

16 Juventino Caminero, “El sistema poético de Unamuno”, Letras de Deusto, 14 (1977), pp. 67-85.

17 Miguel de Unamuno, "Credo Poético", en Poesía Completa, ed. Ana Suárez Miramón, Madrid, Alianza, 1987, I, pp. 53-54 (p. 53). 
La poesía no sólo no se identifica con la música, sino que es algo más. En los versos del Credo Poético, Unamuno plantea un conflicto entre un poema inconsistente ("la columna de humo se disipa entera") y otro, el suyo, de peso ("peso necesitan, en las alas peso").

No es necesario crear un poema lleno de adornos retóricos carentes de todo sentido; el pensamiento poético no debe ser revestido, sino esculpido en el lenguaje ("de escultor y no de sastre es tu tarea"), para sacar de él la idea en toda su belleza; por eso, creo que podemos conjeturar un conocimiento, por parte de Unamuno, del famoso soneto en el que Miguel Ángel describe la actividad de 'extracción' de la idea de la materia del mármol:

Non ha l'ottimo artista alcun concetto c'un marmo solo in sé non circoscriva col suo superchio, e solo a quello arriva la man che ubbidisce all'intelletto ${ }^{18}$.

La investigación, el estudio, no empuja a Unamuno hacia una poesía vacía de musicalidad y de recursos retóricos, sino que lo induce a buscar un ritmo diferente, "a contratiempo" (para recorrer a la expresión rítmicomusical que él mismo utiliza en la composición jId con Dios!, que abre la colección), que sea funcional para expresar el sentido más profundo del texto. Se trata de un detalle muy importante, ya que implica que todas las figuras y las estructuras retóricas con las que el escritor articula sus composiciones son funcionales para la expresión de una idea: el objetivo no es tanto lograr un "vaciado" del ritmo y de la musicalidad, como una cadencia que sea eficaz y adecuada para la expresión de la Idea ${ }^{19}$.

18 Michelangelo Buonarroti, Rime, en Poeti del Cinquecento, ed. Guglielmo Gorni, Milán-Nápoles, Ricciardi, 2001, p. 611. La misma idea se reitera en una carta enviada por el artista a Benedetto Varchi en la que propone una poética de la escultura (y por extensión también de la palabra) que «si fa per arte di levare»; véase Michelangelo Buonarroti, Lettera a Benedetto Varchi, en Le lettere di Michelangelo Buonarroti, Florencia, Le Monnier, 1875, p. 522. Entre los libros de Unamuno se conserva una copia de las Poesie de Buonarroti de 1911 (U/2914 ded. anot.).

19 Biruté Ciplijauskaité, “El sonido es sentido”, en Actas del Congreso Internacional del Cincuentenario de Unamuno, ed. $\mathrm{M}^{a}$ Dolores Gómez Molleda, Salamanca, Univer- 
Si volvemos al texto con esta conciencia, podemos notar fácilmente una serie de repeticiones de palabras o estructuras, todas ricas en significado.

La palabra "torre", por ejemplo, se registra 9 veces en la composición y sirve para identificarla como una interlocutora, o mejor dicho, como espectadora del monólogo del yo poético. En el v. 35, la expresión "Luna compasiva" se inserta para marcar el contraste con el "Sol brutal" del v. 34; con toda probabilidad Unamuno corrige la "l” minúscula original de "luna” para crear una personificación (la Luna) y marcar así una neta oposición de carácter puramente mitológico entre los dos astros, Luna y Sol: un Sol brutal, amenazador, mortífero ("tras de la muerte el Sol brutal se oculta", v. 34), y una Luna reconfortante, sensible, "compasiva Luna" (v. 35), materna ("del sueño madre", v. 36), liberadora ("cuando la Luna / de tus piedras los sueños libertaba / y ellas cedían”, vv. 26-28), que "hace pasar» a través del sueño a lo eterno, de la muerte a la vida, hasta la realización de la redención en la belleza ("despiertas de la muerte y de la vida, / y en lo eterno te sueñas y revives / en tu hermosura", vv. 14-16) que, en mi opinión, constituye el núcleo conceptual, simbólico e ideológico del texto.

Es precisamente este acercamiento-oposición de talante mitológico entre los dos astros, conquistado a lo largo de la escritura y de la reescritura a través de ligeros retoques textuales de los cuales el aparato permite seguir el proceso genético, uno de los motivos figurativos más iluminadores de esta obra. En la segunda estrofa, los dos primeros versos describen la acción de la luz del sol que dora la torre y adormece los recuerdos, mientras que los dos últimos presentan el despertar en la blanca luz de la luna. La imagen se desarrolla en la estrofa sucesiva, donde la torre de oro parece convertirse en un silencioso e invisible centinela: la luz del día salmantino, cortante pero suave, como una sustancia líquida misteriosa, la sumerge y desdibuja los límites. Sin embargo, es la llegada de la oscuridad la que hace que la torre se hunda en su inconsciencia, en los "recuerdos vagarosos" que hasta entonces habían permanecido en estado latente (v. 6).

Sugiero que la idea misma de la liquidez de la luz es la clave para entender el proceso de plasmación de este poema, a partir de la disolución de

sidad de Salamanca, 1989, pp. 429-432. Véase además José de Kock, Cancionero de Miguel de Unamuno, Salamanca, Universidad de Salamanca, 2006, pp. 35-49. 
la terrestre "ley de piedra" (vv. 30 y 37) hasta la conquista de una mágica, pero todavía más espiritual, "eterna / torre de gloria” (vv. 39-40), que la recuperación, conforme a la técnica medieval de las coblas capfinidas (vv. 40-41), reafirma y con fuerzas recobradas de la memoria histórica en el comienzo de la cuarta estrofa: "Torre de Monterrey, soñada torre...".

En cuanto a la elaboración textual me parece conveniente subrayar, en $m s^{l}$, el paso en el v. 10 de "absorve" a "sume". Ya he hablado de los problemas relacionados con la musicalidad, y no volveré a insistir sobre el tema: en este caso, me parece que la variante "te sume" respecto a "se absorve" desplaza sobre la luz la liquidez desprendida que, en un principio, era característica de la torre absorbida por la luz del día: imagen en contraste con las categorías de la robustez (v. $1 \mathrm{~ms}^{\mathrm{l}}$ ) y la solidez (pensemos, por ejemplo, en la referencia a las piedras del v. 27) de la estructura arquitectónica. Luego, en las tres estrofas siguientes, a través de un complejo mecanismo de correcciones, que constituye uno de los nudos genéticos de mayor énfasis también en la historia ideológica y simbólica del texto, vuelve la expresión "en unción" (vv. 13, 18 y 21), en que la luz de la luna parece convertirse en un ungüento mágico (v. 21), capaz de disolver la materia de las cosas.

La ligera variación patente en la repetición "sume/sumerges", se llena entonces de significado, ya que corresponde a un nivel diferente de sentido: el primer verso nos muestra una luna "enajenada” que sufre la acción del sol ("que en sí te sume") y parece desaparecer, hundirse, en la luz que desdibuja los límites; en el segundo caso es la torre, blanca gracias a la luna y luego claramente visible, la que elige hundirse en la inconsciencia ("te sumerges").

Al despertar de la luna corresponde el despertar del ego que siente cómo se avivan los "recuerdos de tras la vida": para enfatizar esta relación, probablemente Unamuno elige la supresión del término "fantasmas" del v. 19, insistiendo, con una repetición significativa, en los "recuerdos" que ya se encontraban en el v. 6: no se puede dejar de señalar que en esos años "recuerdos" era un término querido por Unamuno debido a las largas y articuladas reflexiones en torno a sus memorias que se convertirían en sus Recuerdos de niñez y de mocedad. 
La luz de la luna, a lo largo del desarrollo del poema, manifiesta un poder de figuración alegórica no sólo metafísico, típico de la poesía de la época romántica a partir de la obra de Leopardi, tan apreciada por Unamuno, y que actúa en lo profundo en el plano rítmico-sentimental, como muestra la escansión periódica de mecanismos alocutivos ("Torre de Monterrey, cuadrada torre [...]”, v. 1; “Torre de Monterrey, díme, mi torre [...]", v. 33; “Torre de Monterrey, soñada torre [...]”, v. 40), que no puede dejar de recordar algunos incipit de los Canti, sin duda presentes en la memoria creadora de Unamuno: "O graziosa luna, io mi rammento [...]" (XIV, Alla luna); "Vaghe stelle dell'Orsa, io non credea / Tornare ancor per uso a contemplarvi [...]" (XXII, Le ricordanze); "Che fai tu, luna, in ciel? Dimmi, che fai, / Silenziosa luna?" (XXIII, Canto notturno di un pastore errante dell'Asia); pero también destellos de imágenes como "D'in su la vetta della torre antica, / Passero solitario [...]" (XI, Il passero solitario) $\mathrm{y}$ "[...] siccome torre / In solitario campo, / Tu stai solo, gigante, in mezzo a lei" (XVI Il pensiero dominante).

Esa luz lunar, en cambio, asume un carácter que yo definiría como mágico-psicológico, fruto del recurso a un imaginario folclórico o al replanteamiento de la tradición mitológica clásica y medieval, ambas bien conocidas por nuestro poeta. De esta fuerza cósmica nadie puede escapar, ya que «derrite» la materia de las cosas y consigue liberar el alma secreta oculta bajo la superficie de la realidad. Incluso "las piedras" (vv. 27 y 30) se ven obligadas a ceder ante su poder, arrastradas por un flujo de energía que puede recordar a aquel con el que, en la mitología griega, los poetaschamanes, Orfeo, Anfión y Zeto, desplazaban animales y piedras con la fuerza del pensamiento y del canto.

Si efectivamente en el conjunto de la poesía unamuniana resulta central esta afinidad alegórica entre la luz lunar y el poder de la mente que crea la poesía, todavía asume un mayor valor poetológico la visión, sobre la que se centra la Torre de Monterrey, de un mundo privado de su materialidad mortal y solar; de un mundo lunar, hecho de amor y de compasión. Un mundo compuesto sólo de almas ya liberadas de las ataduras del cuerpo y se confirma así la importancia de la que, a partir de ahora, aparece entre las preocupaciones emblemáticas de la poética unamuniana: la vida después de la muerte: 
La vida es sueño de cierto, pero dinos, desventurado Don Quijote, tú, que despertaste del sueño de tu locura para morir abominando de ella, dinos: ¿no es ensueño también la muerte? ${ }^{20}$

La pregunta que Unamuno dirige a «su» Caballero de la Triste Figura, en 1905 , es probablemente la misma que pocos meses más tarde el yo poético dirige a la torre, ansioso por saber si después de la muerte nos espera realmente un sueńo bańado por la luz de la luna. Incluso en la descripción de la muerte de Ignacio en Paz en la guerra (1897), Unamuno construye una imagen que parece significativamente próxima a la de la Torre de Monterrey: "Sentíase desfallecer por momentos, que se le iba la cabeza, liquidándosele la visión de las cosas presentes, y luego una imersión en un gran sueńo" ${ }^{21}$. Ignacio, como la torre, se sumerge en el sueño de la muerte, donde la visión de la realidad tangible parece licuarse, con una transformación de naturaleza y de estado completamente afín con lo descrito en el poema, en el que el "mundo inmaterial, todo de sueño, / de libertad, de amor, sin ley de piedra” (vv. 29-30) se convierte sin reservas en el "mundo de luz de luna" (v. 31), en el horizonte del cual la liberación desde (y de) la realidad en el sueño coincide con un renacimiento: "Renacer me he sentido a tu presencia”.

Contemplando la eterna torre de piedra que se disuelve gracias a la "unción" mágica de la luz lunar, el yo se pregunta si más allá de la vida, cuando las almas al reunirse formen la "torre de gloria", igualmente eterna (y la labor sobre $m s^{l}$ nos muestra que el escritor agrega y trabaja con precisión justo en la colocación del adjetivo "eterna”) gozarán de la misma libertad del sueño. Con una reanudación que en mi opinión se refiere intencionadamente a un modelo teológico-espiritual formado durante el cristianismo tardoantiguo y medieval, en la "torre de la gloria" domina la imagen de la resurrección post mortem, la brillante conquista de un cuer-

20 Miguel de Unamuno, La vida de don Quijote y Sancho, en Obras completas, III, p. 138.

21 Miguel de Unamuno, Paz en la guerra, ed Francisco Caudet, Madrid, Cátedra, 1999, p. 418; sobre el motivo del sueño en la obra de Unamuno véase R. M. K. Van der Grijp, "Ensueños. Un motivo en el pensamiento de Unamuno", Cuadernos de la Cátedra Miguel Unamuno, 13 (1963), págs. 75-93. 
po glorioso privado de la temporalidad y la corrupción: o, en el sistema metafórico puesto en juego por Unamuno, liberado de la "ley de piedra" derrotada por el "sueño" y el "ensueño".

El poema es, pues, el resultado de una visión y posee un fuerte impacto visual basado en los contrastes entre las luces del día y de la noche. Desde esta perspectiva, tal vez se podría explicar la variante que implica el v. 1 y sobre el cual ya nos hemos detenido para reconstruir la posición cronológica de $m s^{a}$. El aparato nos muestra que la lectura "robusta" se elige en $m s^{l}$, confirmada en $G B$ y Pub, y que, en un primer momento, también se utiliza en $m s^{a}$. Más tarde, tal vez durante proceso de relectura, Unamuno interviene para modificarla: la elimina y sustituye por "cuadrada". Los dos atributos, que remiten a un significado similar, están conectados a la solidez y a la arquitectura de la torre. Tal vez "robusta” permitía dar más fuerza y protagonismo a la intervención de la luz de la luna que, casi por arte de magia, disuelve las piedras de la torre (pensemos, por ejemplo, en la estrofa VI), mientras que "cuadrada" tiene un mayor impacto visual porque proporciona al lector indicaciones sobre la forma de la misma, y por tanto, de alguna manera, le permite participar en la visión del poeta. Sin embargo, como seńaló el mismo García Blanco, la elección de la voz "cuadrada" está motivada principalmente por el deseo de eliminar "la vibrante múltiple del adjetivo robusta, en un verso donde aun hay otras tres" $^{22}$, reduciendo así una desagradable cacofonía del verso.

Otra variante, en el v. 42 -mucho más allá de la mera variación genética de la que da fe-, me parece que ofrece una oportunidad adicional de respiro hermenéutico. La poesía presenta una estructura circular, desde el momento en que el primer verso coincide, al menos en parte, con el último: el v. 1 difiere del v. 41 solo por el atributo que se refiere a la torre ("robusta / soñada") y los vv. 3-4 coinciden con los vv. 43-44. La elección inicial de utilizar en el v. 42 el verbo "desfilar" fue probablemente motivada por la presencia del mismo verbo en el v. 2: la luna había visto pasar, uno tras otro, tanto los hombres y los días (v. 2), como los sueños del poeta (v. 42). Con esta elección Unamuno pretendía, por tanto, reforzar la impresión de simetría entre el íncipit y el éxplicit; en los versos finales, sin

22 Otras reflexiones sobre las variantes señaladas por el estudioso en Manuel García Blanco, op. cit., pág. 79. 
embargo, se esfuerza por dar una mayor importancia a la idea del renacimiento, que, junto con el sueńo de la vida, es el tema central del poema: la torre, un signo del pasado Renacimiento (el período de la historia a que remonta su arquitectura) y al mismo tiempo esperanza del futuro renacer, ha visto renacer los sueños y el propio yo del poeta (como también se dice en el v. 25) ${ }^{23}$. Más tarde, sin embargo, en el momento de su publicación en la revista, Unamuno decide intervenir de nuevo sobre el texto y transforma "renacer" en "madurar". La variante pudo deberse a dos razones diferentes. La primera tiene relación con el sentido del poema, porque la torre se convierte en un testigo no sólo del renacer, sino también del crecimiento psíquico y moral, de la maduración de los sueños del yo, y se convierte en un interlocutor constante en la vida del poeta. La segunda interpretación conduce una vez más a cuestiones rítmico-musicales, acaso porque Unamuno probablemente no estuviera convencido de la repetición tan cercana de "renacer" y "Renacimiento", cuyo contacto es a la vez semántico y semiótico, fónico-gráfico, y no es, como en el caso de "sume / sumerges", suficientemente denso de significado.

La idea, de fuerte potencia simbólica, permanecerá mucho tiempo en la mente y en la escritura poética de Unamuno: diez años después, en un artículo que desde el título, La torre de Monterrey a la luz de la helada, se vincula a la obra que nos ocupa, pondrá de relieve el lazo entre la idea de "renacer" y la imagen alegórica de la torre del Palacio de Monterrey. Contemplando el alto edificio ya no bajo la luz de la madre Luna, capaz de disolver con su luz las piedras sólidas, sino en la fría y cortante luz de la mañana, Unamuno escribirá:

Y esta mi torre de Monterrey me habla de nuestro Renacimiento, del renacimiento español, de la españolidad eterna, hecha piedra de visión, y me dice que me diga espańol, y que afirme que si la vida es sueño, el sueño es lo único que queda $[\ldots]^{24}$.

23 En $m s^{1}$ está claro que Unamuno escribe con tinta la primera versión; introduce luego algunas variantes a lápiz; y finalmente corrige de nuevo con tinta.

24 Miguel de Unamuno, La Torre de Monterrey a la luz de la helada, en Andanzas y visiones españolas, en Obras completas, I, p. 469. 
No solo vuelve aquí la idea, central en el poema, de un Renacimiento individual y al mismo tiempo más marcadamente nacional, sino también la imagen de la vida como sueño.

Un análisis preciso de los papeles de $m s^{a}$ que contienen el poema La torre de Monterrey a la luz de la luna, por lo tanto, permite establecer algunas reflexiones de más amplias vistas acerca de la estructura del «libro de poesía» en el que Unamuno piensa durante esos años. Se sabe que ya estaba planeando entre 1898-1899 la publicación de un librito de poemas con un número muy reducido de composiciones. El proyecto se ampliará al ańo siguiente y en 1900 el escritor anuncia a Clarín que está a punto de publicar una colección titulada Veintisiete poesías, noticia confirmada por el editor madrileño B. Rodríguez Sierra que incluye el libro entre las obras "en preparación" 25 . En la segunda franja del aparato he señalado que, junto al título de la obra, en el f. 54 aparece el número 39. Se trata, en mi opinión, de una numeración alternativa del poema que, en una fase inicial, hasta este momento todavía no señalada por la crítica, tenía que ocupar la posición 39. Si se examina todo el $m s^{a}$, nos daremos cuenta de que todas las composiciones tienen la doble numeración que va, con distintas lagunas, del número 3 de La flor tronchada al 94 de Tendido yo en la cama. Muchas de las lagunas, además, pueden colmarse recurriendo a manuscritos de otros poemas conservados en el Archivo de Salamanca y no incluidos en la colección; el estudio en profundidad de este material, por tanto, permitirá reconstruir un proyecto poético distinto.

Creo, en conclusión, que es necesario preparar una nueva edición de la colección poética de Unamuno que tenga en cuenta la modalidad de reelaboración de las distintas etapas del poemario durante el proceso de creación, y que, por tanto, contemple, en sentido general, el estilo compositivo de Unamuno para "encontrar direcciones, más que límites fijos, de la energía poética" ${ }^{26}$.

25 Manuel García Blanco, op. cit., pp. 9-12.

26 Gianfranco Contini, Saggio di un commento alle correzioni del Petrarca in volgare, en Varianti e altra linguistica, Torino, Einaudi, 1970, p. 5. 